\title{
A 4D Fusion Puzzle
}

\author{
Duco Jansen explains how he, along with engineers at the ITER fusion \\ experiment, will position 1000-ton parts shipped in from the four corners \\ of the globe.
}

By Michael Schirber

$\square$ ast May, the ITER fusion experiment began its assembly phase. Originally known as the International Thermonuclear Experimental Reactor, the project in the south of France is a global effort to confirm the feasibility of a commercial nuclear-fusion reactor. The ITER Collaboration expects to complete construction in 2025, when the first tests with plasma "fuel" will start. But the experiment has already started, says Duco Jansen, ITER's construction manager, as building the machine is a test in and of itself. In the assembly phase, Jansen has his hands full overseeing the assembly hall where giant cryostats, magnets, and other reactor pieces are fitted together one by one. Physics spoke to Jansen to find out what it's like to work on the world's largest puzzle.

\section{All interviews are edited for brevity and clarity.}

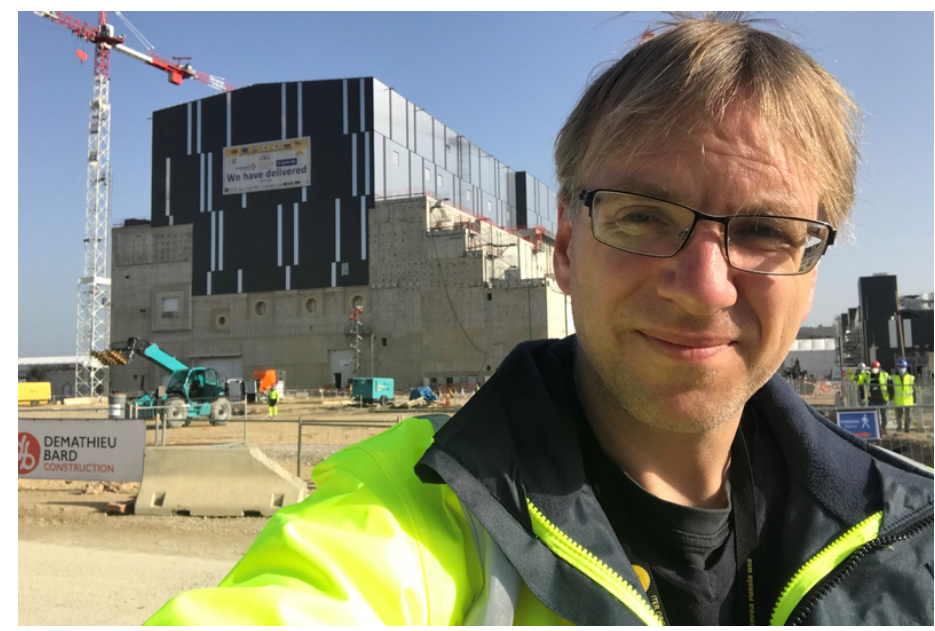

Credit: D. Jansen/ITER

\section{What is it like working at ITER?}

The first thing is the international aspect: 35 countries participate in ITER, which means the people working on the project speak different languages, observe different cultures, and have different ways of doing things. In such an environment, you can't be stuck in the mindset that your way is the only way. You have to be adaptable and flexible.

People often think that a facility's construction is just an intermediate phase before the real experiments start. But the experiment is going on now: The building process is testing whether we can make a tokamak of ITER's size and technical specifications.

\section{Can you explain what you do at ITER?}

My job is to organize the assembly of ITER, which means ensuring that pieces are where they need to be so that everyone can do the work they need to do. Traditionally, procurement for projects of this size is managed centrally, but ITER is different. The member states are providing in-kind contributions, which means they build components in their home countries and then ship them to France for assembly. One benefit of dividing up the fabrication is that fusion technology can be developed in several nations in parallel. But it makes the job of my colleagues and I more complicated. We have to coordinate delivery times, so that a missing piece doesn't cause a delay. We also have to verify that what we receive is in line with the assembly expectations: We don't want a surprise when we "open a box."

\section{How do the pieces get to ITER?}

The very large pieces, called high exceptional loads (HELs), arrive by sea at a port near Marseilles in the south of France. From there, the HELs follow what is known as the "ITER 
This animation depicts the transport of ITER's cryostat base from the assembly hall to the reaction pit. The video is an example of "4D planning," which ITER personnel use to manage the space and time constraints of the reactor's assembly.

Credit: Brigantium Engineering

itinerary," which is a reinforced road that was specially built for ITER. The road is normally open to the public, but the police close it at night when we have a delivery. The itinerary is only 100 kilometers long, but it takes several nights for these big transports to wind their way to the site. Just last month, ITER received its 50th HEL-a cryostat component. Roughly 100 more HELs should arrive over the years to come.

\section{Can you describe how these big pieces come together?} It's like the video game Tetris, where blocks of different shapes are stacked on top of each other. But here we play with million-dollar pieces the size of small buildings while a voice in the back of our heads is saying: "Oh, by the way, we've never installed something like this before." An example is the bottom cylinder of the cryostat-an enormous pancake-shaped slab of stainless steel that was supplied by India and serves as the base of the reactor. It weighs 1250 tons and is ITER's heaviest single piece. It was the first piece that we lowered into the reactor pit in May 2020.

The next big component to go in will be the first "sector module," which is a piece of the doughnut-shaped vacuum vessel where the fusion reactions will take place. The system is 19 meters wide - too big to install whole-so it's been cut up into nine modules. These modules will go in one by one and will be welded together. This assembly will be made more complicated by the cleanliness requirements-we can't leave any dust or grease behind. That is something that keeps me up at night.

\section{If building ITER is an experiment, what will we learn from the process?}

That it is feasible to build an industrial-scale nuclear fusion reactor. There is a joke about nuclear fusion that it is always 30 years away. I don't think that is any longer the case. Previous research in the 1990 s determined that a machine of ITER's size should give a stable fusion process, so I strongly believe that if we build it correctly, it will work.

\section{In your mind, why is ITER worth its estimated 20-billion-euro (\$25-billion) investment?}

Today, we have nuclear fission reactors, fossil-fuel power plants, wind turbines, and solar panels. These choices were given to us through the efforts of previous generations. Investing in ITER could give future generations the option to use nuclear fusion, as well. Whether they choose to do that is up to them, but we want to give them that choice.

\section{Do you see yourself working on fusion reactors in the future?}

This job is a dream, as each technological problem is the first of its kind. I feel privileged to be on the front ranks of this effort. It's hard to know what will happen, but I am convinced that the field has a very attractive future.

Michael Schirber is a Corresponding Editor for Physics based in Lyon, France. 